\title{
Clemens Fuest
}

\section{Finanzpolitik in der neuen Legislaturperiode: Auf wirtschaftliche Erholung und Wachstum setzen!}

\begin{abstract}
Deutschland steht in den kommenden Jahren vor erheblichen ökonomischen Herausforderungen. Die deutsche Wirtschaft hat sich teilweise von der Corona-Krise erholt, aber das Vorkrisenniveau ist bei der Wirtschaftsleistung noch nicht wieder erreicht. Derzeit belasten Lieferprobleme bei vielen Vorprodukten die Konjunktur, die Erholung bleibt also fragil. Hinzu kommt, dass die Pandemie tiefe Spuren hinterlässt. Die Staatsschulden sind im Laufe der Krise erheblich gestiegen. Viele Unternehmen haben durch lange Schließungen Kundschaft, Mitarbeitende und Kapital verloren. Vor allem junge Menschen sind in ihrer Schul- und Berufsausbildung zurückgeworfen worden.
\end{abstract}

Außerdem sind Herausforderungen zu bewältigen, die schon vor der Corona-Pandemie existierten. Der demografische Wandel wird in den nächsten Jahren dazu führen, dass die Zahl der Menschen im Erwerbsalter sinkt. Die schon seit längerer Zeit spürbare Knappheit von Fachkräften wird sich verstärken. Das reduziert die Wachstumspotenziale. Die Alterung der Bevölkerung bringt darüber hinaus erhebliche finanzielle Belastungen für die öffentliche Hand mit sich, vor allem in den Sozialkassen. Die Pandemie hat die Digitalisierung von Wirtschaft und Gesellschaft beschleunigt.

Die Senkung der $\mathrm{CO}_{2}$-Emissionen für Klimaschutz und die Anpassung an den Klimawandel, die Digitalisierung und andere Formen technischen Fortschritts führen zu einem sich beschleunigenden Strukturwandel. Obwohl Deutschland einen sehr stark ausgebauten Sozialstaat hat, steht zu befürchten, dass wirtschaftliche Unterschie-

(C) Der/die Autor:in 2021. Open Access: Dieser Artikel wird unter der Creative Commons Namensnennung 4.0 International Lizenz veröffentlicht (creativecommons.org/licenses/by/4.0/deed.de).

Open Access wird durch die ZBW - Leibniz-Informationszentrum Wirtschaft gefördert.

Prof. Dr. Dr. h. c. Clemens Fuest ist Präsident des ifo Instituts und Professor für Volkswirtschaftslehre, Lehrstuhl für Nationalökonomie und Finanzwissenschaft an der Ludwig-Maximilians-Universität München. de in den kommenden Jahren zunehmen werden, unter anderem deshalb, weil die durch die Pandemie bedingten Ausfälle bei der Schulbildung Kinder und Jugendliche aus bildungsfernen Milieus stärker treffen als andere. Es wird deshalb noch einmal wichtiger, Inklusion und Chancengerechtigkeit zu fördern.

Auch auf europäischer und internationaler Ebene steht Deutschland vor großen Herausforderungen. Die europäische Integration ist ein wichtiger Wohlstandsmotor, aber der Brexit hat die EU massiv geschwächt, und in wichtigen Politikfeldern spielt die EU nicht die Rolle, die sie im Interesse aller Europäer:innen eigentlich spielen sollte. Gleichzeitig wachsen international protektionistische Neigungen und geopolitische Konflikte, vor allem zwischen China und den USA. Nach der Wahl von Joe Biden zum US-Präsidenten hat sich das Verhältnis zu den USA stark verbessert, aber es ist nicht auszuschließen, dass in drei Jahren erneut ein Politiker vom Schlag Donald Trumps gewählt wird. Es wird nicht einfach sein, in diesem Umfeld deutsche und europäische Wirtschaftsinteressen zu wahren.

Dieser Beitrag konzentriert sich auf den Bereich der Finanzpolitik und erläutert, welche Maßnahmen die nächste Bundesregierung ergreifen sollte, um den anstehenden finanzpolitischen Herausforderungen zu begegnen. ${ }^{1} \mathrm{Im}$ Mittelpunkt sollte dabei das Ziel stehen, die wirtschaftliche Erholung nach der Cororna-Krise zu fördern und sie in ein kräftiges und nachhaltiges Wachstum münden zu lassen. Wirtschaftliche Stärke ist keine hinreichende, aber sicherlich eine notwendige Voraussetzung dafür, dass die genannten Herausforderungen erfolgreich bewältigt werden können.

\section{Staatsfinanzen: Wirtschaftliche Erholung und Wachstum priorisieren}

Die Finanzpolitik der nächsten Legislaturperiode steht vor erheblichen Spannungen und Zielkonflikten. Einerseits ist es entscheidend, dass die Finanzpolitik die wirtschaftliche Erholung und die Rückkehr zu stetigem Wirtschaftswachstum unterstützt. Das spricht für steuerliche Entlas-

1 Der Beitrag stützt sich teilweise auf das vom ifo Institut entwickelte wirtschaftspolitische Reformprogramm, das ausführlicher dargestellt wird in: Wirtschaftspolitische Herausforderungen in der Post-MerkelÄra, ifo Schnelldienst 7/2021 (ifo Institut, 2021). 
tungen und mehr öffentliche Investitionen. Andererseits ist die Staatsverschuldung in der Finanzkrise erheblich gestiegen. Die Handlungsspielräume sind also begrenzt. Hinzu kommt, dass einige Parteien fordern, mit Steuern stärker umzuverteilen, vor allem durch die Einführung einer Nettovermögensteuer. Das wiederum würde Investierende abschrecken und das Wirtschaftswachstum beeinträchtigen. Letztlich ist die Gewichtung der verschiedenen Ziele eine politische Wertung. Angesichts der oben beschriebenen Herausforderungen und der nach wie vor fragilen Konjunkturlage spricht aber viel dafür, die Finanzpolitik primär auf die Förderung des Wirtschaftswachstums auszurichten.

Was bedeutet das? Das Wirtschaftswachstum wird vor allem durch die Entwicklung der Beschäftigung und durch Investitionen und Innovationen getrieben. In beiden Bereichen spielen Effizienz und Produktivität sowie die aktuellen Treiber des Strukturwandels eine zentrale Rolle. Eine wachstumsorientierte Finanzpolitik sollte sich darauf konzentrieren, in diesem Sinne Beschäftigung und Investitionen zu fördern. Hohe Beschäftigung und hohe Produktivität sind darüber hinaus entscheidend für Inklusion, also breite Teilhabe am Wirtschaftswachstum.

Wachstum durch mehr Beschäftigung und Arbeitsproduktivität

In den vergangenen beiden Jahrzehnten war die Wirtschaftsentwicklung in Deutschland davon geprägt, dass die Zahl der Erwerbstätigen stetig stieg, unter anderem durch mehr Frauenerwerbstätigkeit und Zuwanderung. Die Arbeitslosigkeit ging zurück, und die Babyboomer-Generation erreichte den Höhepunkt ihrer Produktivität. Das hat das Wirtschaftswachstum gefördert und die Staatsfinanzen entlastet. Dieser Trend wird sich in den kommenden Jahren umdrehen. Die Erwerbsbevölkerung wird älter und schrumpft. Einer sinkenden Zahl von Erwerbstätigen steht eine wachsende Zahl von Ruheständlern gegenüber.

Das hat nicht nur Folgen für die Renten-, Pensions- und Gesundheitskassen. Es ist zu erwarten, dass Schrumpfung und Alterung der Erwerbsbevölkerung auch das Wirtschaftswachstum dämpfen werden. Damit verkleinern sich Handlungsspielräume und Wohlstandspotenziale in allen Bereichen der Gesellschaft. Anders als oft behauptet wird, erschwert ein durch schrumpfende Erwerbsbevölkerung sinkendes Wirtschaftswachstum auch die Chancen, Umwelt- und Klimaschutz erfolgreich voranzutreiben, denn dafür sind Ressourcen erforderlich, vor allem Innovationen und Investitionen.

Ob und in welchem Umfang das Wirtschaftswachstum tatsächlich zurückgeht, hängt allerdings von wirtschafts- und gesellschaftspolitischen Weichenstellungen ab. Wichtig für die Wachstumsentwicklung sind nicht nur die Zahl und die Altersstruktur der Erwerbstätigen, sondern auch die Bereitschaft und Möglichkeit zur Teilnahme am Arbeitsmarkt.

Entscheidend ist darüber hinaus die Produktivität der Beschäftigten. Bessere Ausbildung und Gesundheit, Automatisierung und die Ausstattung mit einem modernen Kapitalstock, intelligenter Einsatz digitaler Techniken und funktionierende Arbeitsmärkte, an denen die Beschäftigten in die richtigen Tätigkeiten gelangen, all dies sind Faktoren, welche die Produktivität erheblich beeinflussen. Wenn hier Verbesserungen erreicht werden, kann das den Rückgang der Erwerbstätigenzahlen zumindest teilweise kompensieren. Das gilt auch für die Zuwanderung von Fachkräften.

Zu den Maßnahmen, welche die Politik hier ergreifen sollte, gehören

- Reformen der Hartz-IV-Regelungen zur Beseitigung der Niedrigeinkommensfalle (Blömer, Fuest und Peichl, 2021),

- eine Einkommensteuerreform, in der das Ehegattensplitting durch ein Realsplitting ersetzt wird, verbunden mit Übergangsfristen für bestehende Ehen,

- ein weiterer Ausbau der Kinderbetreuung,

- eine Förderung der Zuwanderung von Arbeitskräften, unter anderem durch Optimierung des Visumsprozesses und der Terminvergabe, Ausbau der Beschäftigungsmöglichkeiten während der Arbeitssuche (Poutvaara, 2021),

- eine Stärkung der Arbeitsmarktbeteiligung von bereits in Deutschland lebenden Geflüchteten sowie

- Reformen an den Schulen, den Universitäten und in der beruflichen Bildung, die Inklusion und Chancengerechtigkeit fördern. Dazu gehören deutschlandweite Zwischen- und Abschlussprüfungen, Strukturänderungen für einen effizienteren Ressourceneinsatz im Bildungssystem, nachgelagerte Studiengebühren und mehr zertifizierte Weiterbildung (Wössmann, 2021).

Durch ein solches Paket an Reformen könnte Deutschland die wachstumshemmenden Wirkungen des demografischen Wandels zumindest teilweise ausgleichen. Diese Reformen gehören nur zum Teil dem engeren Bereich der Finanzpolitik an, sie haben allerdings alle insofern Relevanz für die Finanzpolitik als sie Implikationen für die öffentlichen Haushalte haben. 


\section{Wachstum durch private und öffentliche Investitionen}

Neben den beschriebenen Maßnahmen zur Stärkung des Arbeitsangebots gilt es private und öffentliche Investitionen zu steigern. Wichtige Treiber für diese Investitionen sind Digitalisierung und Klimaschutz sowie die Anpassung an die Klimaerwärmung. Umstritten ist, was zu tun ist, damit diese Investitionen stattfinden.

Viele Unternehmen stehen vor der Herausforderung, erhebliche Investitionen zu tätigen, um ihre $\mathrm{CO}_{2}$-Emissionen zu senken oder um Chancen der Digitalisierung zu ergreifen. Um das zu ermöglichen und zu fördern, sollten die steuerlichen Rahmenbedingungen für Investitionen und Forschung und Entwicklung in Deutschland verbessert werden. ${ }^{2}$ Das erfordert Steuerentlastungen, zumindest aber die Vermeidung von größeren Steuererhöhungen, insbesondere der Einführung einer Nettovermögensteuer in Deutschland.

In öffentlichen Debatten wird häufig der Eindruck erweckt, die erforderlichen Investition seien in erster Linie staatliche Investitionen. Das ist irreführend. Tatsächlich geht es primär darum, die richtigen Rahmenbedingungen für private Investitionen zu schaffen, im Klimaschutz vor allem durch einen transparenten und umfassenden $\mathrm{CO}_{2}$-Preismechanismus. Es ist darüber hinaus notwendig, den Preismechanismus dort, wo er nicht funktioniert, durch gezielte staatliche Förderungen oder Regulierungen zu ergänzen. Es werden auch öffentliche Investitionen gebraucht, aber sie sind quantitativ zweifellos der deutlich kleinere Teil. ${ }^{3}$

Gerade im Bereich der Dekarbonisierung und der Anpassung an die Erderwärmung besteht eine wichtige Aufgabe der kommenden Bundesregierung darin, die richtige Kombination zwischen der Schaffung guter Rahmenbedingungen für private Investitionen, gezielten Eingriffen durch Regulierungen, Steuern und Subventionen sowie öffentlichen Investitionen zu finden. Das ist deshalb nicht einfach, weil viele der erforderlichen Investitionen an der Grenze zwischen Privatsektor und Staat liegen, etwa bei der Deutschen Bahn, in der Energiewirtschaft oder im Telekommunikationssektor.

Die Abgrenzungsprobleme werden deutlich, wenn man untersucht, um welche Investitionen es sich handelt. Ei-

2 Es liegt auf der Hand, dass es viele andere politisch beeinflusste Standortfaktoren gibt, die hier relevant sind. Dazu gehört z. B. eine preislich wettbewerbsfähige und sichere Energieversorgung.

3 In einer aktuellen Studie über den Bedarf an öffentlichen Investitionen zum Klimaschutz weisen Krebs und Steitz $(2021,2)$ darauf hin, dass „öffentliche Klimainvestitionen nur einen relativ kleinen Teil der gesamten Investitionen in den Klimaschutz darstellen". ne aktuelle Studie der Agora Energiewende (Krebs und Steitz, 2021) errechnet einen zusätzlichen Bedarf an öffentlichen Investitionen in Höhe von 460 Mrd. Euro bis 2030 bzw. 46 Mrd. Euro pro Jahr, also rund 1,3\% des Bruttoinlandsprodukts. Im Zeitraum bis 2025 sind 80 Mrd. davon bereits durch den Bund finanziert. Im Gesamtbetrag in Höhe von 460 Mrd. Euro enthalten sind 200 Mrd. Euro für die Förderung der Gebäudedämmung, also eine Förderung privater Investitionen. Weitere $140 \mathrm{Mrd}$. Euro sollen in Schienenwege, in klimaneutralen sozialen Wohnungsbau, in Fernwärmenetze und in Wasserstoffnetze fließen. Das sind Investitionen, die sinnvoll erscheinen, bei denen aber umstritten sein dürfte, ob der Staat sie finanzieren und durchführen sollte. Das gilt sicherlich für den sozialen Wohnungsbau, bei denen Modelle mit privaten Investierenden und entsprechenden Förderungen und Auflagen effizienter sein dürften. Bei Wasserstoffleitungen und Fernwärmenetzen sind eine zumindest partielle private Finanzierung und eine Steuerung sowie Risikoübernahme durch private Betreibende möglich. Ähnliches gilt für Investitionen im öffentlichen Personennahverkehr, die von der Studie betont werden.

Bei alldem ist die private Finanzierung kein Selbstzweck. Sie kann sogar kontraproduktiv sein, wenn private Investierende nur Finanzierungen bereitstellen, die meist teurer sind als die Finanzierungskosten des Staates, aber keine Risiken übernehmen. Es geht darum, durch privatwirtschaftliche Anreize vor allem bei der Errichtung, aber auch bei den Betreibenden eine höhere Effizienz zu erreichen. Das funktioniert nur, wenn die privaten Investierenden zumindest einen Teil der Risiken tragen.

Unabhängig davon, wie die Rollenverteilung zwischen Staat und privaten Akteur:innen gestaltet wird, liegt eine weitere Aufgabe der kommenden Bundesregierung darin, die Planungs- und Genehmigungsverfahren für Investitionsprojekte in Deutschland zu beschleunigen. Auch dabei muss die Politik mit schwierigen Zielkonflikten umgehen. Die Beteiligung der lokalen Bevölkerung an Entscheidungen über Infrastrukturprojekte wie Stromtrassen oder Bahnstrecken ist ein hohes Gut im demokratischen Rechtsstaat. Wenn die Umsetzung der Projekte dadurch aber um Jahre oder gar Jahrzehnte verzögert wird, ist diese Form der Beteiligung nicht mit den ehrgeizigen Transformationszielen vereinbar wie die Politik sie insbesondere im Klimaschutz anstrebt.

Stehen die Fiskalregeln einer wachstumsorientierten Finanzpolitik im Weg?

Eine wichtige Frage für die Finanzpolitik der kommenden Jahre liegt darin, ob und wie Forderungen nach steuerlichen Entlastungen und mehr öffentlichen Investitionen mit 
den Vorgaben der im Grundgesetz verankerten Schuldenschranke und den europäischen Verschuldungsregeln vereinbar sind. ${ }^{4}$ Die Regelungen der Schuldenbremse stehen höherer Verschuldung für Investitionen jenseits der Defizitgrenzen nicht entgegen, sofern diese Investitionen und deren Finanzierung in öffentlichen Unternehmen stattfinden. Man kann kritisieren, dass es sich hier um Schattenhaushalte handelt, aber angesichts der hohen öffentlichen Aufmerksamkeit für größere Investitionen im Rahmen der Klimapolitik oder der Digitalisierung kann man davon ausgehen, dass diese finanziellen Transaktionen im politischen Prozess hinreichend registriert und diskutiert werden.

Viele Kritiker:innen der Regeln fordern dennoch, die Schuldenbremse zu reformieren, um die darin enthaltenen Verschuldungsspielräume auszuweiten. Viel Unterstützung hat dabei die Idee, zu einer Variante der goldenen Regel zurückzukehren, die eine Kreditfinanzierung öffentlicher Investitionen erlaubt. Eine investitionsorientierte Schuldenregel galt in Deutschland bis zur Einführung der Schuldenbremse im Jahr 2009. Sie hat weder den deutlichen Rückgang öffentlicher Investitionen zwischen 2000 und 2008 noch den stetigen Anstieg der Staatsschuldenquote in den Jahrzehnten davor verhindert. Hinzu kommt, dass die Kreditfinanzierung zumindest klassischer öffentlicher Investitionen etwa im Bereich der Infrastruktur durch die Schuldenbremse kaum eingeschränkt wird, weil die Möglichkeit der Finanzierung in öffentlichen Unternehmen oder Sonderhaushalten besteht. Die Schuldenbremse begrenzt aber in der Tat Spielräume für einen Wachstumsimpuls in Form einer steuerlichen Entlastung für Unternehmen und Beschäftigte. Das gilt auch für steuerliche Zuschüsse für private Klimaschutzinvestitionen wie etwa die Wärmedämmung bei Gebäuden. Hier hilft die Einführung einer investitionsorientierten Schuldenregel jedoch nicht weiter, weil auch sie für diese Ausgaben keine Kreditfinanzierung vorsieht. ${ }^{5}$

Sowohl die Steuersenkungen als auch die Zuschüsse für die Wärmedämmung haben insofern Charakteristika öffentlicher Investitionen als sie heute die öffentlichen Haushalte belasten und man dadurch in Zukunft auf einen Nutzen in Form höherer Wirtschaftsleistung hofft. Das verweist auf eine entscheidende Schwäche investitionsbasierter Schuldenregeln - die Abgrenzung investiver von nicht investiven Ausgaben ist schwierig.

4 Eine aktuelle Analyse der finanziellen Spielräume in den kommenden Jahren unter Berücksichtigung der Schuldenbremse und der europäischen Fiskalregeln bieten Feld et al. (2021).

5 Es werden Verschuldungsspielräume erhöht soweit bestehende Investitionen, die bislang nicht kreditfinanziert sind, künftig durch Kredite finanziert werden. Ob und in welchem Umfang dadurch Mittel frei werden, hängt unter anderem davon $a b$, ob die Investitionsregel Abschreibungen auf den öffentlichen Kapitalstock berücksichtigt.
Angesichts dieser Schwierigkeiten könnte man auf die Idee kommen, die Schuldenbremse ganz abzuschaffen. Das wäre jedoch ein Fehler. Die Schuldenbremse stellt für die mittelfristige Finanzpolitik in Deutschland einen wichtigen Anker dar, der Glaubwürdigkeit stärkt und die Politik einem heilsamen Druck aussetzt, Prioritäten zu setzen. Ein weniger weitreichender Eingriff würde darin bestehen, ähnlich wie in den Jahren vor 2016 einen Übergangspfad zu definieren, der in den nächsten Jahren vorübergehend zusätzliche Finanzierungsspielräume eröffnet (Feld et al., 2021). Das würde allerdings eine Grundgesetzänderung erfordern, für die keine Mehrheit in Sicht ist. Außerdem wäre es unklug, über eine Aufweichung der Schuldenbremse zu verhandeln, ohne dass ein überzeugendes und hinreichend konkretes finanzpolitisches Konzept vorliegt, das den zusätzlichen Finanzbedarf überzeugend begründet und eingrenzt. Ein solches finanzpolitisches Konzept sollte folgende Elemente enthalten:

1. Eine umfassende Steuerreform für Angestellte und Unternehmen, mit der die Rahmenbedingungen für Arbeitsangebot, Investitionen und Innovationen verbessert werden. Elemente dieser Reform könnten die bereits erwähnten Reformen im Niedrigeinkommensbereich und in der Ehegattenbesteuerung, verbesserte steuerliche Abschreibungen für Investitionen ${ }^{6}$ sowie eine erweiterte steuerliche Verlustverrechnung sein.

2. Der Solidaritätszuschlag wird für alle Steuerzahlenden abgeschafft, dafür wird der Einkommensteuertarif neugestaltet. In diese Reform können verteilungspolitische Zielsetzungen eingebracht werden. Zu berücksichtigen sind dabei insbesondere die Folgen für Arbeitsangebotsanreize und für Investitionsanreize der Personengesellschaften, die der Einkommensteuer unterliegen.

3. Ein Programm für öffentliche Investitionen und Förderungsmaßnahmen für private Investitionen in Klimaschutz, Anpassung an den Klimawandel und Digitalisierung. Bei Fördermaßnahmen für private Investitionen ist zu bedenken, dass Investitionsanreize primär durch die $\mathrm{CO}_{2}$-Bepreisung entstehen sollten und eine Förderung nur dann infrage kommt, wenn die Wirkungen der Bepreisung nicht ausreichen oder der Preismechanismus wegen bestehender Regulierungen oder sonstiger Marktfriktionen nicht wirkt.

Wenn ein überzeugendes finanzpolitisches Konzept für die Stärkung des Wachstums und die Bewältigung der Transformation zu Digitalisierung und Klimaneutralität vorliegt,

6 Die ökonomischen und fiskalischen Wirkungen beschleunigter steuerlicher Abschreibungen werden analysiert in Dorn et al. (2021) 
kann diskutiert werden, welche fiskalischen Auswirkungen sich daraus ergeben und in welchem Verhältnis die erforderlichen Mittel durch Ausgabenkürzungen, Steuererhöhungen bzw. den Abbau von Steuersubventionen und Kreditfinanzierung aufgebracht werden sollen. In diesem Schritt kann auch geklärt werden, ob die Spielräume, welche die Schuldenbremse bietet, ausreichen, oder ob vorübergehend größere Spielräume erforderlich sind.

\section{Literatur}

Blömer, M., C. Fuest und A. Peichl (2019), Raus aus der Niedrigeinkommensfalle(!) Der ifo-Vorschlag zur Reform des Grundsicherungssystems, ifo Schnelldienst, 72(4), 34-43.
Dorn, F., C. Fuest, F. Neumeier und M. Stimmelmayr (2021), Wie beeinflussen Steuerentlastungen die wirtschaftliche Entwicklung und das Steueraufkommen?, ifo Schnelldienst, August, 3-11.

Feld, L., L. Nöh, W. H. Reuter und M. Yeter (2021), Von der Corona-bedingten Schuldenaufnahme zur Wiedereinhaltung der Schuldenbremse, Sachverständigenrat zur Begutachtung der gesamtwirtschaftlichen Entwicklung, Arbeitspapier, 1, Mai.

ifo Institut (2021), Wirtschaftspolitische Herausforderungen in der PostMerkel-Ära, ifo Schnelldienst, 74(7).

Krebs, T. und J. Steitz (2021), Öffentliche Finanzbedarfe für Klimainvestitionen im Zeitraum 2021-2030, Forum New Economy Working Paper, 3

Poutvaara, P. (2021), Zuwanderung von Fachkräften fördern, ifo Schnelldienst, 74(7), 11-14.

Wössmann, L. (2021), Bildung für Wirtschaftswachstum und Chancengleichheit, ifo Schnelldienst, 74(7), 15-17. 